\title{
GA-A21711
}

\section{THE TEXT NEUTRAL LITHIUM BEAM EDGE DENSITY DIAGNOSTIC}

\author{
by \\ A.M. HOWALD, J.M. McCHESNEY, and W.P. WEST
}

This is a preprint of a paper to be presented at the

Tenth Topical Conference on High Temperature

Plasma Diagnostics, May 8-12, 1994, Rochester, New York, and to be printed in the Proceedings.

Work supported by

U.S. Department of Energy

Grant No. DE-FG03-92ER54150

\section{GENERAL ATOMICS PROJECT 3937 \\ JULY 1994}




\section{DISCLAIMER}

This report was prepared as an account of work sponsored by an agency of the United States Government. Neither the United States Government nor any agency thereof, nor any of their employees, make any warranty, express or implied, or assumes any legal liability or responsibility for the accuracy, completeness, or usefulness of any information, apparatus, product, or process disclosed, or represents that its use would not infringe privately owned rights. Reference herein to any specific commercial product, process, or service by trade name, trademark, manufacturer, or otherwise does not necessarily constitute or imply its endorsement, recommendation, or favoring by the United States Government or any agency thereof. The views and opinions of authors expressed herein do not necessarily state or reflect those of the United States Government or any agency thereof. 


\section{DISCLAIMER}

Portions of this document may be illegible in electronic image products. Images are produced from the best available original document. 


\begin{abstract}
A fast neutral lithium beam has been installed on the TEXT tokamak for Beam Emission Spectroscopy (BES) studies of the edge plasma electron density profile. The diagnostic was recently upgraded from ten to twenty spatial channels, each of which has two detectors, one to measure lithium beam signal and one to monitor plasma background light. The spatial resolution is $6 \mathrm{~mm}$, and the temporal resolution is designed to be as high as $10 \mathrm{~ms}$ for studies of transient events including plasma density fluctuations. Initial results are presented from the ten-channel system: Edge electron densities unfolded from the LiI $\left(2 \mathrm{~s}^{2} \mathrm{~S}-2 \mathrm{p}^{2} \mathrm{P}\right) 670.8 \mathrm{~nm}$ emission profile have the same general time dependence as the line-averaged density measured by microwave interferometry.
\end{abstract}




\section{INTRODUCTION}

Atom and ion beams of many different types have been used as diagnostics of fusion plasma parameters. One subset of these beam diagnostics is that for which the measured quantity is light emitted by beam particles. High-power heating beams and low power diagnostic beams have both been used, and the general technique is called beam emission spectroscopy (BES). Beams of neutral lithium atoms are a common choice since they are relatively easy to produce, lithium is a benign plasma contaminant, and the lithium resonance transition line lies in the visible part of the spectrum. Fast neutral lithium beams have been used as probes of plasma edge densities in ASDEX, ${ }^{1}$ TEXTOR, $^{2}$ and $\mathrm{CHS},{ }^{3}$ of local magnetic field angle in TEXT, ${ }^{4}$ and of plasma density fluctuations in DIII-D. ${ }^{5}$ Neutral lithium beams have also been proposed as targets for charge exchange recombination spectroscopy (CERS) of plasma impurity ions. ${ }^{6}$

This paper describes a neutral lithium beam installed on TEXT as a diagnostic of the edge density profile and of density fluctuations. A neutral lithium beam is injected radially into the plasma on the tokamak mid-plane, and is viewed from below. The $\mathrm{Li}^{+}$ ion source and neutralizer are described in Ref. 7 . The neutral beam intensity is 1-2 $\mathrm{mA}$ at a beam energy of $20-30 \mathrm{keV}$, and the diameter of the beam in the plasma is $2.5 \mathrm{~cm}$.

When the lithium beam enters the plasma it is predominantly in the ground Li $2 s^{2} \mathrm{~S}$ state. Collisions between the beam and plasma electrons and ions populate higher energy levels in the beam atoms and attenuate the beam due to ionizing collisions. This is illustrated in Fig. 1, which shows the populations of the $\operatorname{Li} 2 s^{2} \mathrm{~S}, \mathrm{Li} 2 \mathrm{p}^{2} \mathrm{P}$, and $\mathrm{Li}(\mathrm{n}=3)$ levels calculated for a TEXT-like plasma. This calculation is done by a computer program which solves a set of coupled differential equations for the various $\operatorname{Li}(\mathrm{nl})$ states, using a data base of measured or calculated cross sections for collisional and radiative transitions among the various states. 8 The intensity light from the LiI $\left(2 s^{2} S-2 p^{2} P\right)$ 
resonance transition is directly proportional to the population of atoms in the $2 \mathrm{P}$ state and is the measured quantity. The useful diagnostic range of the beam in the plasma is on the order of 10 or $15 \mathrm{~cm}$, limited by beam attenuation and by saturation of the ratio of the populations of atoms in the ${ }^{2} \mathrm{~S}$ and ${ }^{2} \mathrm{P}$ states.

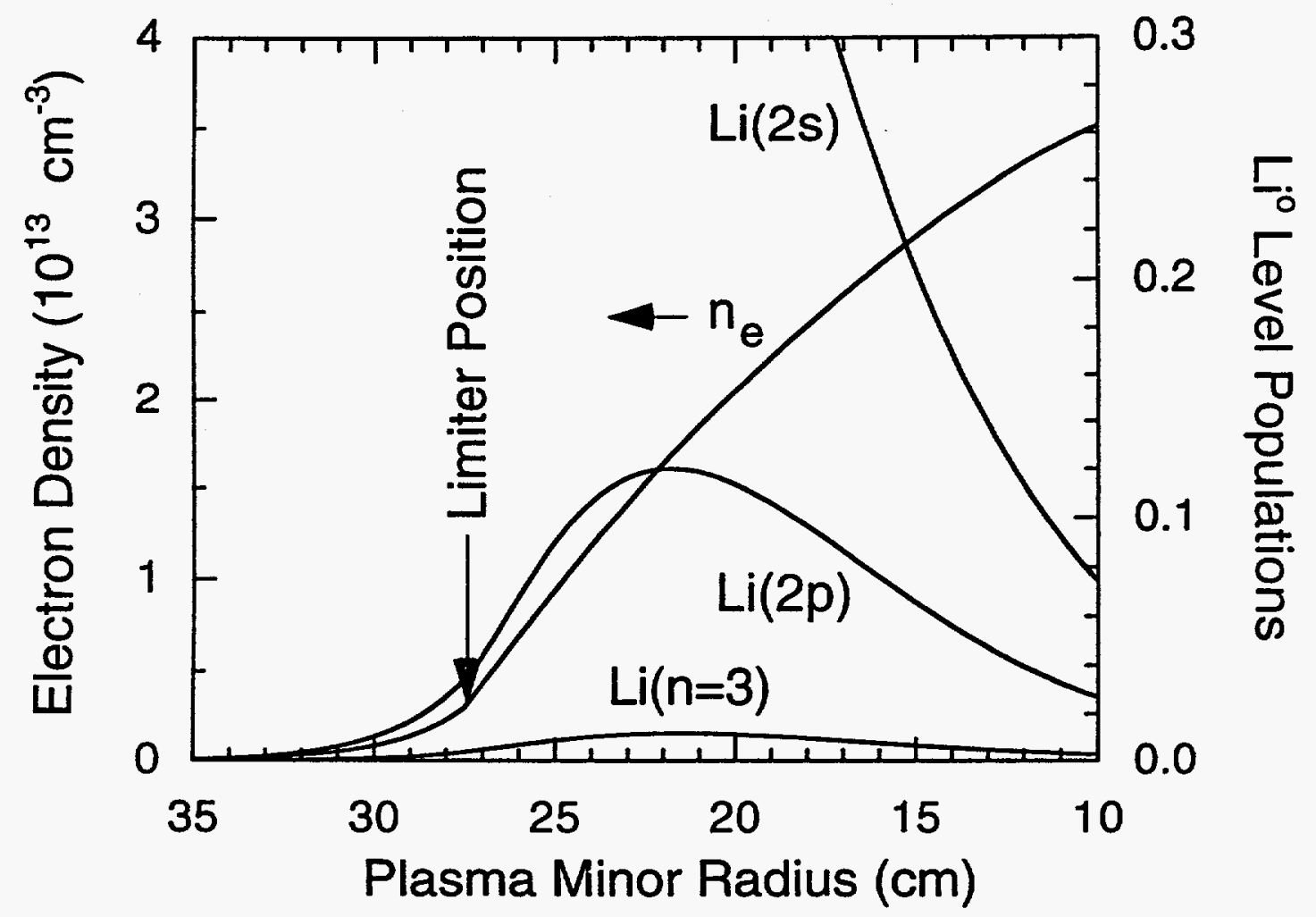

FIG. 1. $\mathrm{Li} 2 \mathrm{~s}^{2} \mathrm{~S}, \mathrm{Li} 2 \mathrm{p}^{2} \mathrm{P}$, and $\mathrm{Li}(\mathrm{n}=3)$ level populations, normalized to the initial beam intensity, calculated for a $25 \mathrm{keV} \mathrm{LiO}$ beam traversing a plasma with a size and density representative of the TEXT tokamak. $\mathrm{Li}\left({ }^{2} \mathrm{~S}-2 \mathrm{P}\right)$ resonance radiation at $670.8 \mathrm{~nm}$, proportional to the $\mathrm{Li} 2 \mathrm{p}^{2} \mathrm{P}$ level population, is the measured quantity.

The $\mathrm{Li} 2 \mathrm{P}$ level population and the $670.8 \mathrm{~nm}$ emission intensity are not directly proportional to the local plasma density, due to attenuation of the beam and the finite lifetime of the $2 \mathrm{P}$ level, so the electron density profile must be "unfolded" from the fluorescence profile. The unfolding process consists of finding a putative electron density profile for which the $\mathrm{Li} 2 \mathrm{p}^{2} \mathrm{P}$ population profile matches the measured $670.8 \mathrm{~nm}$ emission profile. Because of the large amount of data to be analyzed (up to 50,000 profiles per shot), a new faster technique was developed to do this calculation. The density profile is 
assumed to be the sum of a smooth, slowly varying average density plus rapidly varying fluctuations. The slowly varying part of the density profile is determined by a fitting routine and the differential equation for the evolution of the $2 \mathrm{p}^{2} \mathrm{P}$ level population is linearized and solved algebraically for the fluctuations. This algorithm will be described in detail in a separate paper. ${ }^{9}$ 


\section{DETAILS OF THE OPTICAL DETECTION SYSTEM}

A schematic of the experimental optical detection system is shown in Fig. 2. It is designed to have a high photon collection efficiency, uniform spatial sensitivity (within each channel), and to discriminate between lithium light and plasma background light. Lithium $670.8 \mathrm{~nm}$ light emitted from the beam is focused by a system of two lenses and an optical wedge onto a fiber optic assembly. The lens L1 subtends a solid angle of $1.8 \times$ $10^{-2}$ sr. The wedge, which bends the optical axis of the system, is required by physical constraints imposed by the location of the port and adjacent diagnostics. Separate multielement collection (L1) and focusing (L2) lenses minimize the aberrations in the system, reducing cross-talk between adjacent channels due to blurring of the image.

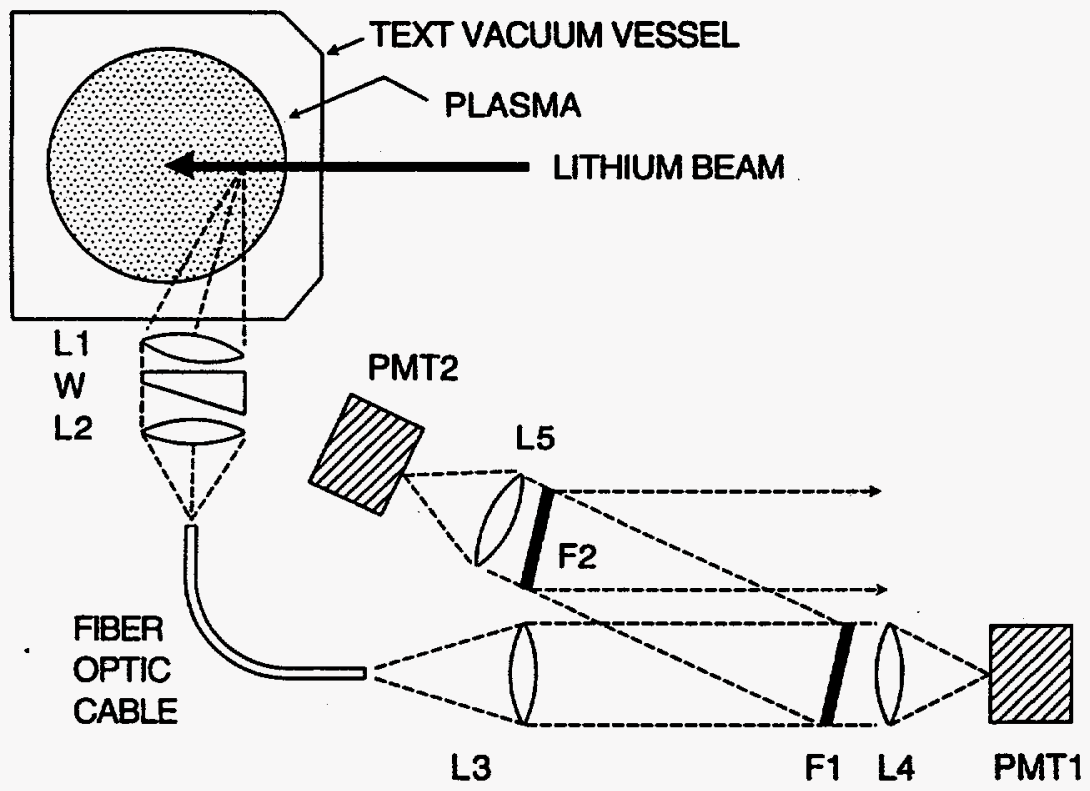

FIG. 2. A schematic of the optical system. Lens $L 1$, wedge $W$, and lens $L 2$ focus light from the lithium beam onto an array of 20 fiber optic bundles. The remainder of the figure shows the optics for one of the 10 spatial channels. Light from the fiber bundle is collimated by lens L3. Interference filter F1 passes light at $670.8 \mathrm{~nm}$ (bandwidth $=1.0 \mathrm{~nm}$ ) and reflects light at other wavelengths. Light that gets through F1 is focused by lens L4 onto photomultiplier PMT1. This light has two components: $\mathrm{Li}\left({ }^{2} \mathrm{~S}-2 \mathrm{P}\right)$ signal and plasma background. Light reflected from $\mathrm{F} 1$ goes to $\mathrm{F} 2$, an interference filter centered at $685 \mathrm{~nm}$ (bandwidth = $3.0 \mathrm{~nm}$ ). Light that gets through $\mathrm{F} 2$ is focused by lens L5 onto photomultiplier PMT2. This light has only a plasma background component. 
The fiber assembly consists of twenty individual bundles. The remainder of Fig. 2 is representative of one of the twenty spatial channels, each of which has two photomultipliers. Each fiber bundle cable has a $3 \times 12 \mathrm{~mm}$ rectangular cross section at the tokamak end, where they butt up against each other with no gap separating adjacent bundles. Each fiber bundle collects light from a section of beam $6 \mathrm{~mm}$ in radial extent and up to $24 \mathrm{~mm}$ in toroidal extent. At the detector end, each fiber bundle has a round cross section, and each spatial channel consists of a two-color polychromator.

Lens L3 collimates the light exiting the fiber bundle, which is focused, after passing through interference filter F1, onto the detector PMT1. The pass band of filter F1 has a central wavelength centered on the (Doppler shifted) $670.8 \mathrm{~nm}$ lithium line, and a FWHM of $1.0 \mathrm{~nm}$. Because of the finite solid angle subtended by lens L1, the $670.8 \mathrm{~nm}$ lithium line has a Doppler broadened width of $0.3 \mathrm{~nm}$ at the filter. The fiber assembly is being changed to one in which the individual fiber bundles will have a rectangular cross section at the detector end as well, since the PMT photocathodes onto which the bundle ends are imaged are rectangular. In addition, the individual fibers in the bundles will be randomized, for reasons explained below.

Filter F2 has a central wavelength of $685 \mathrm{~nm}$ and a FWHM of $3 \mathrm{~nm}$, and is used to monitor plasma background light. Filter F1 reflects $>90 \%$ of light within the pass band of filter F2. Light that passes through filter F2 is focused onto detector PMT2. PMT1 and PMT2 are Hamamatsu R636 gallium-arsenide photomultipliers with approximately 10\% quantum efficiency at these wavelengths.

This experimental design has evolved to its present form in order to reduce or eliminate two of the major sources of experimental error that were discovered while analyzing early data: plasma background light and detector non-uniformity.

Plasma Background Light. Plasma continuum light near $670.8 \mathrm{~nm}$ passes through interference filter F1 (Fig. 2), so that the light measured by photomultiplier PMT1 is a sum of two parts, a "signal" component from the lithium beam and a "background" 
component from plasma continuum, notably molecular hydrogen light from the plasma edge. In Figs. 3(a) and 3(b), which compare the measurements for two similar shots with and without the lithium beam, the signal to background ratio is approximately 5:1, although it varies with plasma radius and average density. For slow time scales and reproducible plasmas, the "background" measured on PMT1 with the lithium beam off [Fig. 3(b)] can be subtracted from the "signal plus background" measured on PMT1 with the lithium beam on [Fig. 3(a)]. For fast time scales or transient events, however, the background intensity will not be reproducible from shot to shot, and its subtraction can introduce significant error. This is the reason for the second photomultiplier PMT2, which is designed to measure the plasma "background" simultaneously with the "signal plus background", at a slightly different wavelength. As shown in Fig. 3, the "background" measured by PMT2 [Fig. 3(c)] when the lithium beam is on is similar to what would have been measured by PMT1 [Fig. 3(b)] with the lithium beam off. To the extent that transient features in plasma background near $670.8 \mathrm{~nm}$ (F1 and PMT1) are similar to those at $685 \mathrm{~nm}$ (F2 and PMT2), this method will allow the subtraction of the "background", including transients, from the "signal plus background".

Detector Non-Uniformity. Each of the 20 detector channels is designed to measure the integral of the light intensity profile over a $6 \mathrm{~mm}$ long section of the beam path. According to the manufacturer, the photomultiplier tubes used for this experiment do not have uniform sensitivity over their active area. ${ }^{10}$ This sensitivity can vary by more than a factor of 2 in $1.5 \mathrm{~mm}$, the distance corresponding to the $6 \mathrm{~mm}$ width of the channel in the plasma. This can introduce errors in the experimental determination of the integrals of the ( $\left.{ }^{2} \mathrm{~S}-2 \mathrm{P}\right)$ light over the $6 \mathrm{~mm}$ widths of the spatial channels, since the light intensity can vary significantly over this distance in the plasma (Fig. 1). If, for example, the brightest part of a channel is imaged onto the part of the detector with the lowest sensitivity, this light will be under-represented in the integral and the signal from that detector will be too low. To circumvent this problem, a new non-coherent fiber assembly is being assembled 
with randomized fibers in each of the bundles. This way, any part of the image of a particular channel (formed by lens L2 in Fig. 2) will be distributed over the entire active area of the photomultiplier, and all sections of the image will be detected with a uniform (average) sensitivity.

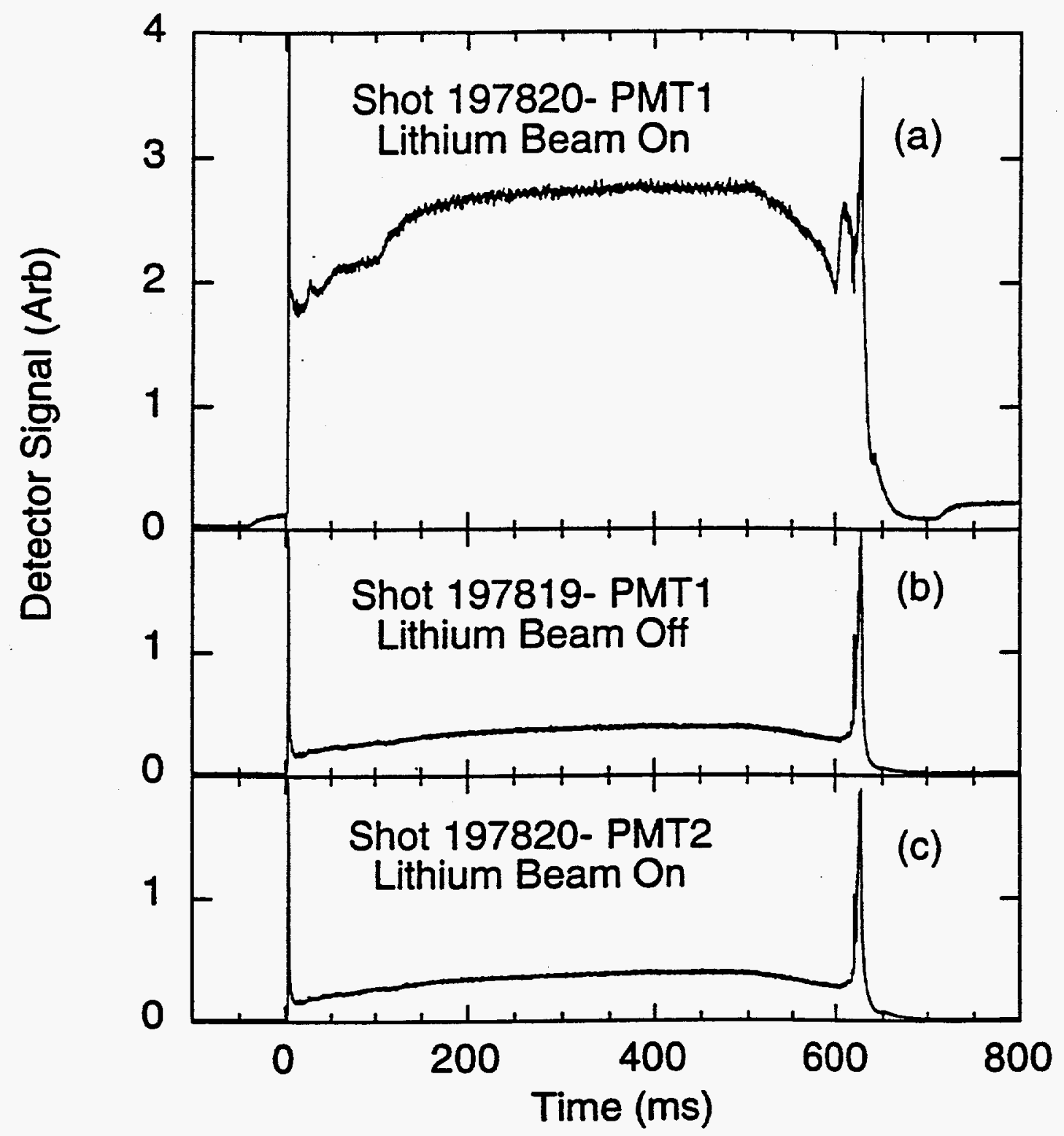

Fig. 3. Raw data from one spatial channel. (a) PMT1 and lithium beam on. This trace has two components, $\mathrm{Li}\left({ }^{2} \mathrm{~S}-2 \mathrm{P}\right)$ signal and plasma background. (b) PMT1 and lithium beam off. This trace was measured on a different shot than (a) and has only a plasma background component. (c) PMT2 and lithium beam on. This trace was measured on the same shot as (a) and has only a plasma background component. 


\section{RESULTS}

Figure 4 shows the electron density as a function of time determined from the lithium beam data at one position inside the plasma for the shots shown in Fig. 3. This data was taken with the ten-channel system and with the old (non-randomized) fiber assembly. Because of the systematic errors in the intensity profiles introduced by the nonuniform sensitivities of the photomultipliers, this data was analyzed using only the first part of the two-part density unfolding algorithm: A least-squares fitting procedure was used to find the smooth electron density profile that best fit the measured lithium beam intensity profile. With twenty spatial channels and the new fiber bundle in place, differences between the measured and fit intensity profiles will be further analyzed to determine the density profile, including fluctuations, with higher resolution. The points in Fig. 4 are plotted every $10 \mathrm{~ms}$, and each point is calculated from lithium beam data which has been averaged for $2 \mathrm{~ms}$. The edge electron density calculated in this manner from the lithium beam qualitatively reproduces the major features visible in the line-averaged density measured by the microwave interferometer. 


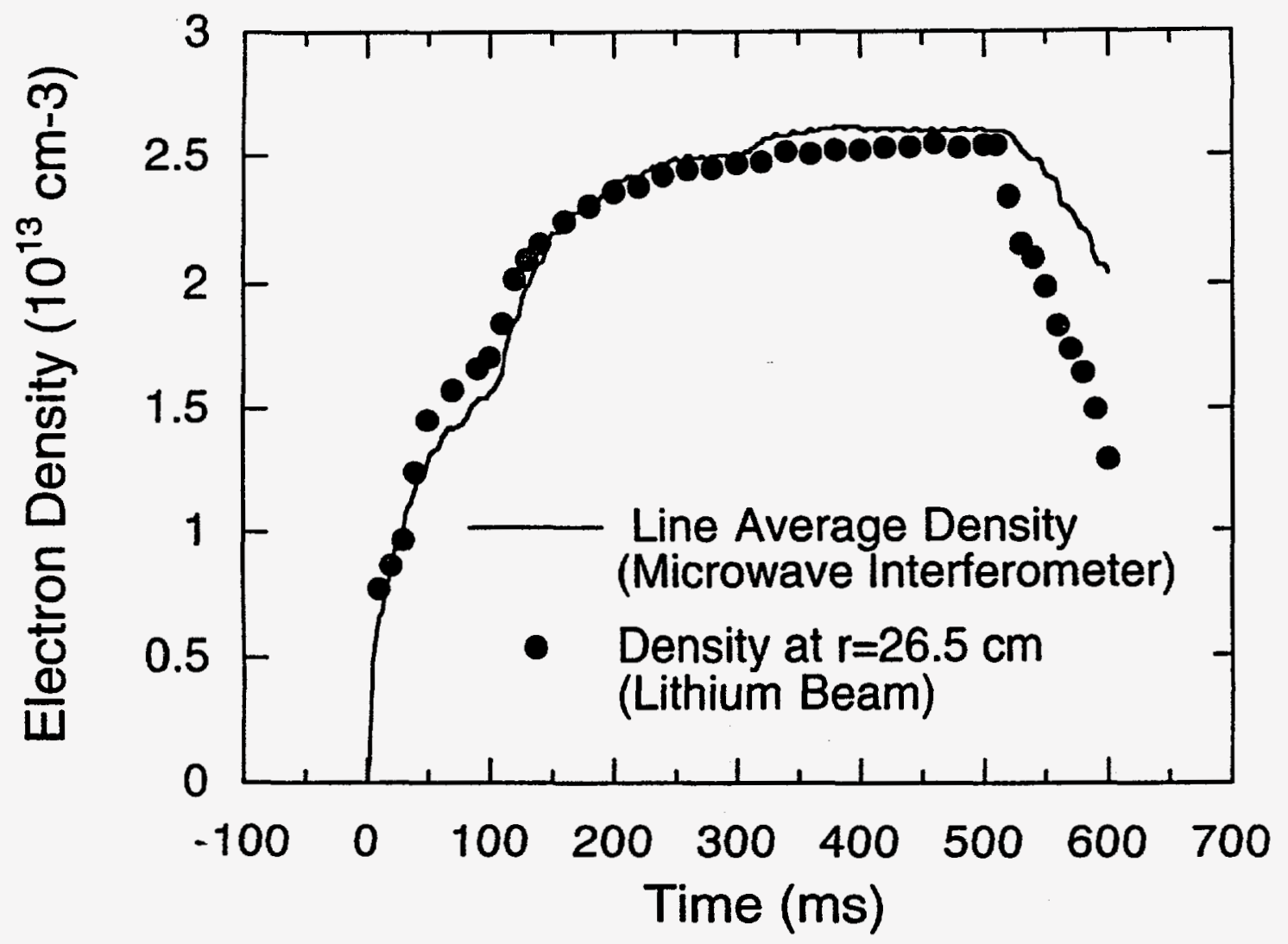

FIG. 4. The edge density unfolded from the lithium beam data reproduces the major features seen in the line average density measured by a microwave interferometer. Increasing the number of detector channels from 10 to 20 and installing the new fiber optic assembly described in the text should improve the resolution and decrease the errors in the lithium beam measurements. 


\section{REFERENCES}

1G.K. McCormick and Z.A. Pietrzyk, J. Nucl. Mater. 162-164, 264 (1989).

${ }^{2}$ E. Wolfrum, F. Aumayr, D. Wutte, H.P. Winter, E. Hinze, D. Rusbuildt, and R.P.

Schorn, Rev. Sci. Instrum. 64, 2285 (1993).

${ }^{3}$ M. Ueda, H. Iguchi, S. Sasaki, and J. Fujita, J. Nucl. Mater. 196-198, 923 (1992).

${ }^{4}$ L.K. Huang, M. Finkenthal, D. Wróblewski, H.W. Moos, and W.P. West, Phys. Fluids B 2, 809, (1990).

5D.M. Thomas, Paper F3, these proceedings.

6R.P. Schorn, E. Wolfrum, F. Aumayr, E. Hinze, D. Rusbüldt, and

H. Winter, Nucl. Fusion 32, 351 (1992).

7W.P. West, D.M. Thomas, E.S. Ensberg, J.S. deGrassie, and J.F. Baur, Rev. Sci. Instrum. 57, 1552 (1986).

${ }^{8}$ R.K. Janev, J.J. Smith, F. Aumayr, D. Wutte, M. Schneider, H.P. Winter, and J. Schweinzer, IAEA Report INDC(NDS)-267 (1993).

9J.M. McChesney, to be published.

${ }^{10} \mathrm{An}$ example of this spatial non-uniformity for side-on photomultiplier tubes is given in the Hamaamatsu Photomultiplier Tube catalog. 


\section{ACKNOWLEDGMENT}

The authors gratefully acknowledge the cooperation of the staff of the TEXT tokamak at the Fusion Research Center in Austin, Texas, and in particular many helpful discussions with Drs. Roger Bengtson and William Rowan. This is a report of work supported by the U.S. Department of Energy under Contract No. DE-FG03-92ER54150. 